\title{
Robust Online State of Charge Estimation of Lithium-Ion Battery Pack Based on Error Sensitivity Analysis
}

\author{
Ting Zhao, ${ }^{1,2}$ Jiuchun Jiang, ${ }^{1,2}$ Caiping Zhang, ${ }^{1,2} \mathrm{Kai} \mathrm{Bai}^{3}$ and $\mathrm{Na} \mathrm{Li}^{3}$ \\ ${ }^{1}$ National Active Distribution Network Technology Research Center (NANTEC), Beijing Jiaotong University, \\ No. 3 Shang Yuan Cun, Haidian District, Beijing 100044, China \\ ${ }^{2}$ Collaborative Innovation Center of Electric Vehicles in Beijing, Beijing 100044, China \\ ${ }^{3}$ State Grid Jibei Electric Power Co., Ltd., Research Institute, No. 1 Dizang'an Nanxiang Fuxingmenwai Street, Beijing 100045, China
}

Correspondence should be addressed to Jiuchun Jiang; jcjiang@bjtu.edu.cn

Received 29 May 2015; Accepted 20 September 2015

Academic Editor: Xiaosong $\mathrm{Hu}$

Copyright (C) 2015 Ting Zhao et al. This is an open access article distributed under the Creative Commons Attribution License, which permits unrestricted use, distribution, and reproduction in any medium, provided the original work is properly cited.

\begin{abstract}
Accurate and reliable state of charge (SOC) estimation is a key enabling technique for large format lithium-ion battery pack due to its vital role in battery safety and effective management. This paper tries to make three contributions to existing literatures through robust algorithms. (1) Observer based SOC estimation error model is established, where the crucial parameters on SOC estimation accuracy are determined by quantitative analysis, being a basis for parameters update. (2) The estimation method for a battery pack in which the inconsistency of cells is taken into consideration is proposed, ensuring all batteries' SOC ranging from 0 to 1 , effectively avoiding the battery overcharged/overdischarged. Online estimation of the parameters is also presented in this paper. (3) The SOC estimation accuracy of the battery pack is verified using the hardware-in-loop simulation platform. The experimental results at various dynamic test conditions, temperatures, and initial SOC difference between two cells demonstrate the efficacy of the proposed method.
\end{abstract}

\section{Introduction}

As one of the most important performance parameters of traction batteries, real-time SOC estimation of battery becomes necessary in the field of application of batterydriven electric vehicles. Commonly used SOC estimation methods for single battery are as follows $[1,2]$ : ampere hour integration method; open circuit voltage method using the corresponding relation between the open circuit voltage and SOC; the algorithm based on electric circuit models or electrochemical models and the typical methods which are Kalman filters and methods based on some observer; estimation using fuzzy logic or methods of machine learning. Since the dynamic battery shows high nonlinearity, these mentioned methods have their own defects: ampere hour integration method has to know the exact SOC initial value and can bring accumulative error; the open circuit voltage scheme has strict requirements on measuring conditions and update of OCV curves can be a difficult task, and it is therefore not suitable for electric vehicle during driving and would better be used as auxiliary correction means; the accuracy of estimation method based on circuit model depends on the model parameters; however, adaptation of the model parameters over the battery lifetime to any given battery aging state or environment requires relatively complex algorithms and is practical for only quite simple models. Some researches based on Kalman filter have achieved online estimation [2-4], but online realization is at the expense of additional computing power and its robustness needs to be concerned; the electrochemical method cannot be applied into operation due to the complexity of the model itself [5]; fuzzy control or vector machine algorithm requires a large number of sample data to train the model and also is no longer applicable for aged battery [6].

A large number of batteries are connected in series and parallels when used in electric vehicles to reach the corresponding level of voltage, power, and energy [3]. Therefore, accurate estimation of state of charge for a battery pack is remaining challenging. Typical SOC calculation method for battery packs [7-10] takes the group as a large battery and 


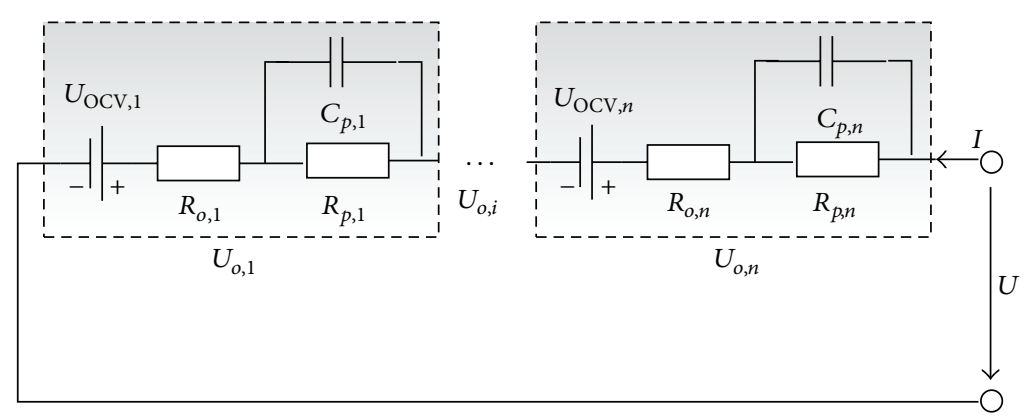

FIGURE 1: The model of series-connected battery pack.

then applies one of the methods described in previous sections, while inconsistent characteristics of cell capacity and resistance cannot be ignored or lead to low management efficiency. Some methods calculate the mean SOC of a battery pack based on mean model or difference model considering cells discordance $[8,11-13]$; others estimate the SOC of every cell in battery pack using common calculation methods and then obtain the pack SOC $[7,14]$. From the above references, methods considering multiple cells are more reliable for battery pack SOC estimation. However, in practical application of pack SOC estimation, the operation conditions, measurement accuracy, and computation of the algorithm need to be considered in integration.

A key contribution of this paper is solving problems in practical application of SOC estimation. In the paper, SOC estimation error model is established, where the crucial parameters on SOC estimation accuracy are determined by quantitative analysis. Battery pack SOC estimation method considered cell inconsistency is on the basis of cell SOC. And single cell SOC is estimated through PI observer combined ampere hour integration method. Also, online identification of the model parameters can make sure of the applicability of the algorithm under different operating conditions during the battery life cycle. In addition, a hardware-in-loop platform is built to fulfill real-time estimation. The verification results of the two series-connected battery packs under different circumstances indicate that this method has a high accuracy and achieves better convergence and stability.
The remainder of this paper is organized as follows: Section 2 analyzes the SOC estimation error theoretically. Section 3 builds series-connected battery pack model, gives the SOC definition, and describes online model parameters update. Our validation results and discussions for different cases of battery pack are presented in Section 4. Section 5 summarizes the conclusions of the paper.

\section{Sensitivity Analysis of SOC Estimation}

2.1. The Proposed SOC Definition for a Battery Pack. For series-connected battery packs, we adopt the model presented in Figure 1, which is a group model connected with many first-order Thevenin models in series. The definition for SOC of battery strings is given in formula (1), where $Q_{\max }^{B}$ denotes the maximum available capacity of the pack and $Q_{\text {rem }}^{B}$ denotes residual capacity, namely, the maximum discharge capacity for the group. As long as we know SOC value and the maximum available capacity of each cell in packs, group SOC will be achieved. Through the proposed SOC pack model, battery pack SOC estimation with inconsistency among cells can be considered on the one hand; on the other hand, as long as the group SOC, namely, $\mathrm{SOC}^{B}$, ranges from $0 \%$ to $100 \%$, all single cell SOC will change in the range of $0 \%$ to $100 \%$. Thus overcharging or overdischarging for the battery can be effectively avoided, and it also can provide data support and ensure secure use of the battery pack:

$$
\begin{aligned}
\operatorname{SOC}^{B} & =\frac{Q_{\text {rem }}^{B}}{Q_{\max }^{B}} \times 100 \% \\
& =\frac{\min \left(Q_{\max }[1] \operatorname{SOC}[1], \ldots, Q_{\max }[n] \mathrm{SOC}[n]\right)}{\min \left(Q_{\max }[1] \operatorname{SOC}[1], \ldots, Q_{\max }[n] \operatorname{SOC}[n]\right)+\min \left(Q_{\max }[1](1-\operatorname{SOC}[1]), \ldots, Q_{\max }[n](1-\operatorname{SOC}[n])\right)} \\
& =\frac{Q_{\max }[i] \operatorname{SOC}[i]}{Q_{\max }[i] \operatorname{SOC}[i]+Q_{\max }[j](1-\operatorname{SOC}[j])} \times 100 \% .
\end{aligned}
$$

2.2. SOC Estimation Error Modeling. Based on the definition of SOC for battery strings in Section 2.1, error of group SOC comes from single cell SOC error. Therefore, error analysis in this part aims for single cells. When the battery is in a different environment or state, the model parameters are also not the same. Through analyzing impact factors of SOC estimation error and influencing degree of each impact factor, SOC estimation accuracy can be improved effectively. Consequently, SOC estimation error formula is deduced mathematically based on the first-order Thevenin battery model $[15,16]$. In 
the formula, parameters in the coefficient matrices $\widetilde{\mathbf{A}}, \widetilde{\mathbf{B}}, \widetilde{\mathbf{C}}, \widetilde{\mathbf{D}}$ are estimation value and have difference with the true value matrices $\mathbf{A}, \mathbf{B}, \mathbf{C}, \mathbf{D}$, where $\mathbf{A}=\left[\begin{array}{cc}-1 / R_{p} C_{p} & 0 \\ 0 & 0\end{array}\right], \mathbf{B}=\left[\begin{array}{c}1 / C_{p} \\ 1 /\left(3600 \cdot C_{n}\right)\end{array}\right]$, $\mathbf{C}=\left[\begin{array}{ll}1 & a_{i}\end{array}\right]$, and $\mathbf{D}=R_{o}$. The actual and estimated state equations of battery are listed in (2), error is expressed in (3), and the final SOC error expression is represented in (9), where $\dot{\mathbf{x}}=\left[\begin{array}{c}\dot{U}_{p} \\ \text { SOCC }\end{array}\right], y=U_{o}-b_{i}, u=I$, and OCV $=a_{i} \cdot \mathrm{SOC}+b_{i}$. Consider

$$
\begin{aligned}
& \dot{\mathbf{x}}=\mathbf{A} \mathbf{x}+\mathbf{B} u, \\
& y=\mathbf{C x}+\mathbf{D} u, \\
& \dot{\widetilde{\mathbf{x}}}=\widetilde{\mathbf{A}} \widetilde{\mathbf{x}}+\widetilde{\mathbf{B}} u+\mathbf{L}\left(U_{o}-\widetilde{U}_{o}\right), \\
& \widetilde{y}=\widetilde{\mathbf{C}} \widetilde{\mathbf{x}}+\widetilde{\mathbf{D}} u, \\
& \mathbf{e}=\widetilde{\mathbf{x}}-\mathbf{x} \\
& \dot{\mathbf{e}}=\dot{\widetilde{\mathbf{x}}}-\dot{\mathbf{x}}=\widetilde{\mathbf{A}} \widetilde{\mathbf{x}}+\widetilde{\mathbf{B}} u+\mathbf{L}\left(U_{o}-\widetilde{U}_{o}\right)-[\mathbf{A} \mathbf{x}+\mathbf{B} u] \\
& =\widetilde{\mathbf{A}} \widetilde{\mathbf{x}}-\mathbf{A} \mathbf{x}+(\widetilde{\mathbf{B}}-\mathbf{B}) u \\
& +\mathbf{L}\left[\mathbf{C} \mathbf{x}-\widetilde{\mathbf{C}} \widetilde{\mathbf{x}}+(\mathbf{D}-\widetilde{\mathbf{D}}) u+b_{i}-\widetilde{b}_{i}\right], \\
& \dot{\mathbf{e}}=\widetilde{\mathbf{A}} \widetilde{\mathbf{x}}-\widetilde{\mathbf{A}} \mathbf{x}+(\widetilde{\mathbf{A}}-\mathbf{A}) \mathbf{x}+(\widetilde{\mathbf{B}}-\mathbf{B}) u \\
& -\mathbf{L}\left[\widetilde{\mathbf{C}} \widetilde{\mathbf{x}}-\widetilde{\mathbf{C}} \mathbf{x}+(\widetilde{\mathbf{C}}-\mathbf{C}) \mathbf{x}+(\widetilde{\mathbf{D}}-\mathbf{D}) u+b_{i}-\widetilde{b}_{i}\right] \\
& =\widetilde{\mathbf{A}}(\widetilde{\mathbf{x}}-\mathbf{x})+\Delta \mathbf{A} \cdot \mathbf{x}+\Delta \mathbf{B} \cdot u \\
& -\mathbf{L}\left[\widetilde{\mathbf{C}}(\widetilde{\mathbf{x}}-\mathbf{x})+\Delta \mathbf{C} \cdot \mathbf{x}+\Delta \mathbf{D} \cdot u-\Delta b_{i}\right] \\
& =(\widetilde{\mathbf{A}}-\mathbf{L} \widetilde{\mathbf{C}}) \mathbf{e}+(\Delta \mathbf{A}-\mathbf{L} \cdot \Delta \mathbf{C}) \cdot \mathbf{x} \\
& +(\Delta \mathbf{B}-\mathbf{L} \cdot \Delta \mathbf{D}) \cdot u-\mathbf{L} \cdot b_{i}, \\
& \dot{\mathbf{e}}=\left[\begin{array}{c}
\dot{e}_{U_{p}} \\
\dot{e}_{\mathrm{SOC}}
\end{array}\right] \\
& =\left[\begin{array}{cc}
-\frac{1}{\widetilde{R}_{p} \widetilde{C}_{p}}-L_{1} & -L_{1} \widetilde{a}_{i} \\
-L_{2} & -L_{2} \widetilde{a}_{i}
\end{array}\right]\left[\begin{array}{c}
e_{U_{p}} \\
e_{\mathrm{SOC}}
\end{array}\right] \\
& +\left[\begin{array}{cc}
-\Delta \frac{1}{R_{p} C_{p}} & -L_{1} \cdot \Delta a_{i} \\
0 & -L_{2} \cdot \Delta a_{i}
\end{array}\right]\left[\begin{array}{c}
U_{p} \\
\mathrm{SOC}
\end{array}\right] \\
& +u\left[\begin{array}{c}
\Delta \frac{1}{C_{p}}-L_{1} \cdot \Delta R_{o} \\
\frac{1}{3600} \cdot\left(\Delta \frac{1}{C_{n}}\right)-L_{2} \cdot \Delta R_{o}
\end{array}\right]-\left[\begin{array}{c}
L_{1} \cdot \Delta b_{i} \\
L_{2} \cdot \Delta b_{i}
\end{array}\right] .
\end{aligned}
$$

$$
\begin{aligned}
e_{\mathrm{SOC}}= & \underbrace{e_{\mathrm{SOC}}(0) e^{-L_{2} \widetilde{a}_{i} t}+L_{2} u\left[-\widetilde{R}_{p}^{2} \widetilde{C}_{p} \cdot e^{\left(-1 / \widetilde{R}_{p} \widetilde{C}_{p}\right) t}+R_{p}^{2} C_{p} \cdot e^{\left(-1 / R_{p} C_{p}\right) t}\right]}_{\text {transient error }} \\
& +\underbrace{\frac{1}{L_{2} \tilde{a}_{i}}\left[u\left(\frac{1}{3600} \cdot \Delta \frac{1}{C_{n}}-L_{2} \cdot\left(\Delta R_{o}+\Delta R_{p}\right)\right)-L_{2} \cdot \Delta b_{i}-L_{2} \cdot \Delta a_{i} \cdot \mathrm{SOC}\right]}_{\text {steady-state error }}=e_{\mathrm{SOC}} 1+e_{\mathrm{SOC}} 2 .
\end{aligned}
$$


TABLE 1: SOC estimation error with different parameter error for a battery with the capacity of 8.8 Ah.

\begin{tabular}{|c|c|c|c|c|c|c|c|c|}
\hline \multirow{2}{*}{ Factors } & \multicolumn{8}{|c|}{ Factor error } \\
\hline & $0.2 \%$ & $0.5 \%$ & $1 \%$ & $2 \%$ & $5 \%$ & $10 \%$ & $20 \%$ & $30 \%$ \\
\hline \multicolumn{9}{|c|}{$e_{\mathrm{SOC}}(\%)$} \\
\hline$\Delta Q$ & -0.0000037 & -0.0000092 & -0.0000183 & -0.0000362 & -0.0000879 & -0.000168 & -0.000308 & -0.000426 \\
\hline$\Delta R_{\text {total }}$ & 0.0296 & 0.074 & 0.148 & 0.296 & 0.74 & 1.48 & 2.96 & 4.44 \\
\hline$\Delta a_{i}$ & -0.2 & -0.5 & -1 & -2 & -5 & -10 & -20 & -30 \\
\hline$\Delta b_{i}$ & 1.45 & 3.62 & 7.24 & 14.5 & 36.2 & - & - & - \\
\hline
\end{tabular}

For expression $e_{\mathrm{SOC}}(0) e^{-L_{2} \tilde{a}_{i} t}$, because the maximum value of $e_{\mathrm{SOC}}$ is one and the value of $L_{2} \widetilde{a}_{i}$ is not too small, thus the value of $e^{-L_{2} \tilde{a}_{i} t}$ will soon decline over time. Of course the expression $e_{\mathrm{SOC}}(0) e^{-L_{2} \widetilde{a}_{i} t}$ can be ignored. As for other part of the transient error $L_{2} u\left[-\widetilde{R}_{p}^{2} \widetilde{C}_{p} \cdot e^{\left(-1 / \widetilde{R}_{p} \widetilde{C}_{p}\right) t}+R_{p}^{2} C_{p} \cdot e^{\left(-1 / R_{p} C_{p}\right) t}\right]$, it will get small as time goes by and tend to zero gradually after $360 \mathrm{~s}$ (6 min), while the value of SOC decreases only about $1.4 \%$ during this period of time. Transient error can be ignored considering the actual application and the analysis. And the example for transient error will be displayed in Figure 1.

On the other hand, from the expression of the steadystate error can we see that SOC estimation error is brought by the error of capacity, total internal resistance, and OCV. The variable $e_{\mathrm{SOC}} 2$ is expressed in (10) and according to the actual simulation data, the sensitivity of each factor for SOC estimation will be analyzed furtherly. Consider

$$
\begin{aligned}
e_{\mathrm{SOC}} 2= & \frac{i}{L_{2} \cdot \widetilde{a}_{i}} \cdot \frac{1}{3600} \cdot \Delta\left(\frac{1}{Q}\right)-\frac{i}{\widetilde{a}_{i}} \cdot \Delta R_{\text {total }} \\
& -\frac{\Delta a_{i} \cdot \operatorname{SOC}(t)}{\widetilde{a}_{i}}-\frac{\Delta b_{i}}{\widetilde{a}_{i}}=e_{1}-e_{2}-e_{3}-e_{4} .
\end{aligned}
$$

2.3. Quantified Analysis. The SOC estimation error is divided into transient and steady-state error from the analysis in Section 2.2 and both will be discussed, respectively. Equation (11) is used to calculate and analyze transient SOC error, where resistance $R_{p}$, capacitance $C_{p}$, observer coefficient $L_{2}$, and input current $u$ are all based on the simulation value or test data and they are assigned the value of 0.6 milliohms, $80000 \mathrm{~F}, 10$, and DST operating current condition, respectively, in this case. SOC transient error calculation results are presented in Figure 2, in which the black line represents the SOC transient error with $2 \%$ polarization resistance and capacitance error while the red line is on behalf of SOC transient error of $5 \%$ polarization resistance and capacitance error; that is, the values of $R_{p}$ and $C_{p}$ are assigned to $1.02 R_{p}$, $1.02 C_{p} / 1.05 R_{p}, 1.05 C_{p}$. Although the initial SOC transient error is large, it will hold the tendency of decline over time and decrease to almost zero within $360 \mathrm{~s}$. And the value of SOC only reduced about $1.4 \%$ during this time; thus it can be ignored in practical application. Consider

$$
\begin{aligned}
& e_{\mathrm{SOC}} 1 \\
& \quad=L_{2} u\left[-\widetilde{R}_{p}^{2} \widetilde{C}_{p} \cdot e^{\left(-1 / \widetilde{R}_{p} \widetilde{C}_{p}\right) t}+R_{p}^{2} C_{p} \cdot e^{\left(-1 / R_{p} C_{p}\right) t}\right] .
\end{aligned}
$$

In terms of SOC steady-state error, conditions like different capacity, different charge, or discharge current rates are discussed, respectively.

(1) $\mathrm{A} \mathrm{Li}(\mathrm{NiCoMn}) \mathrm{O}_{2}$ battery with the capacity of $8.88 \mathrm{Ah}$ is used for analysis. According to the parameter identification results for this cell, taking SOC as $55 \%$ as an example, total internal resistance equals 25 milliohms, the slope $a_{i}$ of the OCV-SOC line is 0.5 , and intercept is 3.62 ; the value of current is $1 / 3 \mathrm{C}$; observer coefficient is taken to be 10 according to the simulation. Considering the error caused by $\Delta a_{i}$ alone, since the SOC ranges from 0 to 1 , thus SOC maximum value is taken as 1 in this case. The results of $e_{1}, e_{2}, e_{3}, e_{4}$ with different variable errors are shown in Table 1:

$$
\begin{aligned}
e_{\mathrm{SOC}}(t)= & \frac{2.96}{10 \cdot 0.5} \cdot \frac{1}{3600} \cdot \Delta\left(\frac{1}{Q}\right)-\frac{2.96}{0.5} \cdot \Delta R_{\text {total }} \\
& -\frac{\Delta a_{i} \cdot \operatorname{SOC}(t)}{0.5}-\frac{\Delta b_{i}}{0.5} \\
= & 1.64 \times 10^{-4} \Delta\left(\frac{1}{Q}\right)-5.92 \cdot \Delta R_{\text {total }}-2 \Delta a_{i} \\
& \cdot \operatorname{SOC}(t)-2 \Delta b_{i} .
\end{aligned}
$$

(2) $\mathrm{A} \mathrm{C} / \mathrm{LiMn}_{2} \mathrm{O}_{4}$ battery with the capacity of $90 \mathrm{Ah}$ is considered as an example. Like in case one, total internal resistance is 1.5 milliohms when $55 \%$ SOC is taken as an example, the slope $a_{i}$ of the OCV-SOC line is 0.4 , and intercept $b_{i}$ is 3.786; current value is $1 / 3 \mathrm{C}$ and observer coefficient is taken to be 10 through simulation. The results of $e_{1}, e_{2}, e_{3}, e_{4}$ with different variable errors are shown in Table 2.

(3) In terms of the same battery as in case (2), considering the situations with different charge and discharge rates, since only $e_{1}, e_{2}$ are affected by the rate of current, we only discuss these two. Comparison results are listed in Table 3 and we can conclude $e_{1}$ is very small and tends to zero. On the basis of this, SOC steady-state error under DST condition is presented in Figure 3 which only considers the influence of internal resistance error $e_{2}$.

Both theoretical analysis and calculation results show that the influencing degree of four impact factors for SOC estimation error is closely related to the battery capacity and charging or discharging current rate. The degree of influence under the same error range ranks as follows: $\Delta a_{i}>\Delta b_{i}>$ $\Delta R_{\text {total }}>\Delta Q$. However, in a practical situation, when OCVSOC curve is linearized, error of $a_{i}$ (the slope of the line) may reach several tens of percent and $b_{i}$ (the intercept of the line) 
TABLE 2: SOC estimation error with different parameter error for a battery with the capacity of $90 \mathrm{Ah}$.

\begin{tabular}{|c|c|c|c|c|c|c|c|c|}
\hline \multirow{2}{*}{ Factors } & \multicolumn{8}{|c|}{ Factor error } \\
\hline & $0.2 \%$ & $0.5 \%$ & $1 \%$ & $2 \%$ & $5 \%$ & $10 \%$ & $20 \%$ & $30 \%$ \\
\hline \multicolumn{9}{|c|}{$e_{\mathrm{SOC}}(\%)$} \\
\hline$\Delta Q$ & -0.0000046 & -0.000012 & -0.000022 & -0.000045 & -0.00011 & -0.00021 & - & - \\
\hline$\Delta R_{\text {total }}$ & 0.0225 & 0.0563 & 0.113 & 0.225 & 0.563 & 1.125 & 2.25 & 3.375 \\
\hline$\Delta a_{i}$ & -0.196 & -0.49 & -0.98 & -1.96 & -4.9 & -9.8 & -19.6 & -29.4 \\
\hline$\Delta b_{i}$ & 1.893 & 4.733 & 9.465 & 18.93 & 43.25 & - & - & - \\
\hline
\end{tabular}

TABLE 3: SOC estimation error with different parameter error and rate for a battery with the capacity of $90 \mathrm{Ah}$.

\begin{tabular}{ccccc}
\hline \multirow{2}{*}{ Factor error } & \multicolumn{5}{c}{ Current rate } \\
& $0.5 \mathrm{C}$ & $1 \mathrm{C}$ & $1.5 \mathrm{C}$ & $2 \mathrm{C}$ \\
\hline \multicolumn{5}{c}{$e_{\mathrm{SOC}}(\%)$} \\
$2 \%$ & -0.00007 & -0.00014 & -0.00020 & -0.00027 \\
$5 \%$ & -0.00017 & -0.00033 & -0.00050 & -0.00066 \\
$10 \%$ & -0.00032 & -0.00063 & -0.00095 & -0.0013 \\
$20 \%$ & -0.00058 & -0.00116 & -0.00174 & -0.00231 \\
$\Delta R_{\text {total }}$ & & & & \\
$2 \%$ & 0.3375 & 0.6750 & 1.013 & 1.350 \\
$5 \%$ & 0.8438 & 1.688 & 2.531 & 3.375 \\
$10 \%$ & 1.688 & 3.375 & 5.063 & 6.750 \\
$20 \%$ & 3.375 & 6.750 & 10.13 & 13.50 \\
$30 \%$ & 5.063 & 10.13 & 15.19 & 20.25 \\
\hline
\end{tabular}

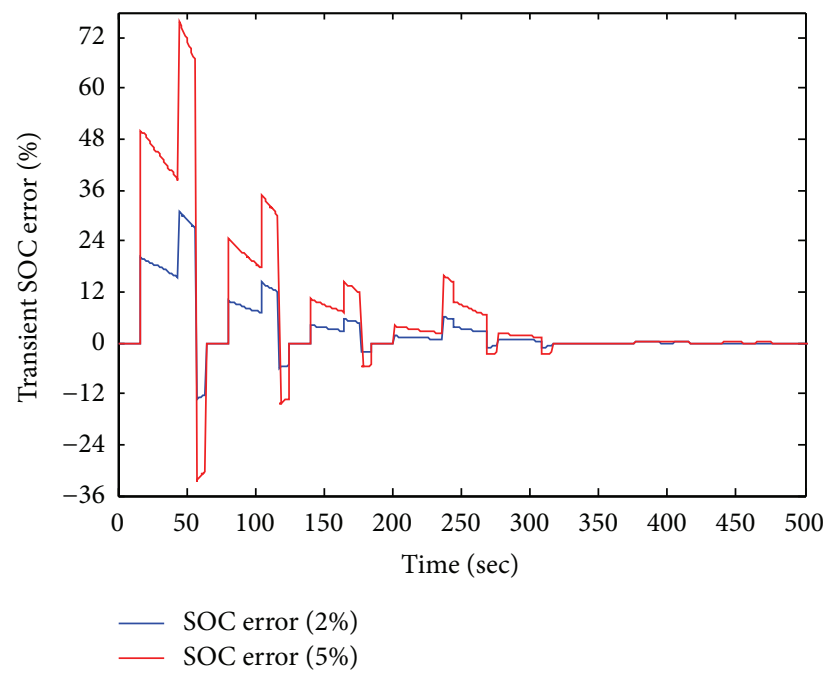

FIGURE 2: SOC estimation transient error with different polarization resistance error under DST condition with two cycles.

error reaches one-thousandth over aging. Therefore, the OCV-SOC curve has a great influence on SOC estimation error and the accurate measurement of OCV is very helpful to reduce the estimation error. Besides, the effect degree of total internal resistance error on estimation is following and the error of capacity is affected the least, while as charging and discharging current rates get large, SOC estimation error

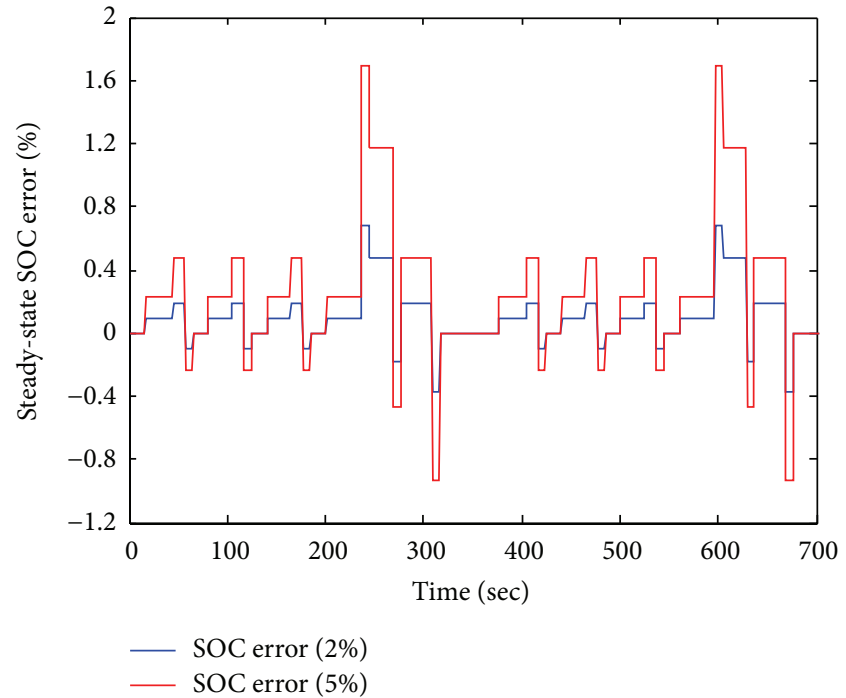

FIgURE 3: SOC estimation steady-state error with different resistance error under DST condition.

caused by these two factors will become larger and the impact of total internal resistance error is especially marked. Based on the quantitative calculation and analysis above, we can see it is of profound significance for SOC estimation to improve the accuracy of model parameters.

\section{Battery Pack SOC Estimation}

3.1. The Proposed SOC Estimation Method for a Battery Pack. The group SOC estimation method adopted in this paper is listed in Figure 4. The battery model parameters of each cell are obtained by online identification. SOC of each cell is estimated with PI observer estimation combined ampere hour integration method; then group SOC can be calculated according to the SOC pack model.

3.2. Online Update of Battery Model Parameters. With the increase of actual use time of the battery and the change in temperature of the environment, battery model parameters like the internal resistance and OCV-SOC curve will also change. In practice, if battery model parameters are not updated, it will bring battery model error and lead to SOC estimation error furtherly. To deal with this problem, the recursive least square method will be employed for online battery model parameters identification. Achievement 


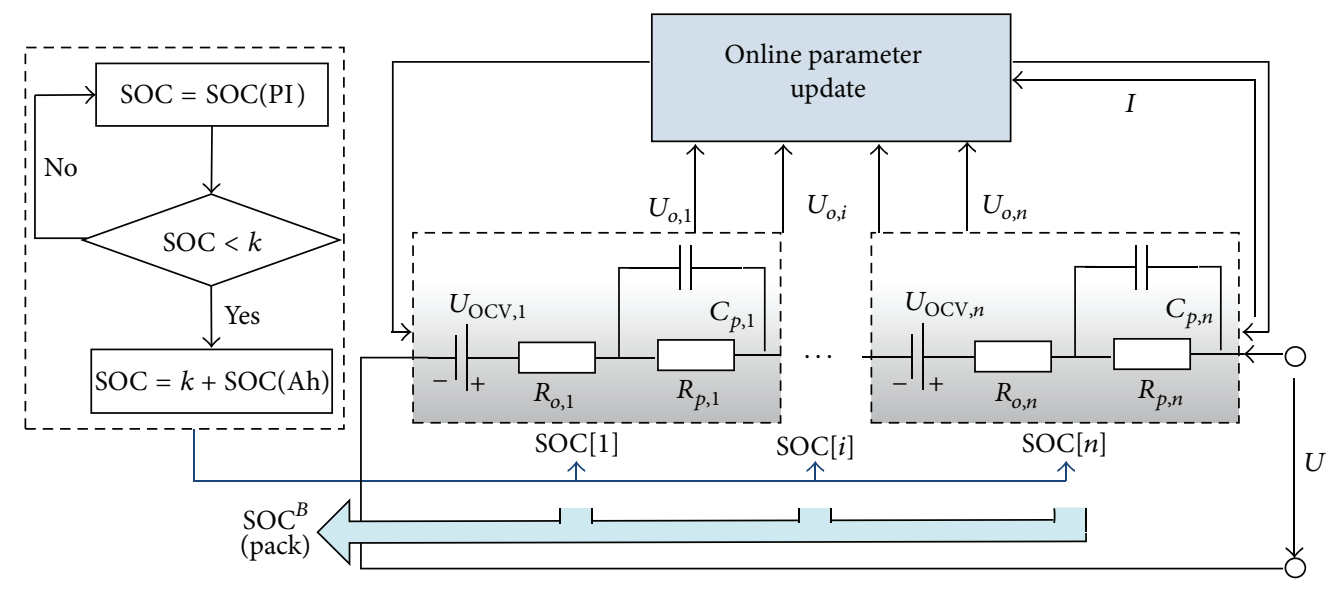

FIGURE 4: The proposed group SOC estimation method.

of real-time update reduces parameter error and thereby increases the SOC estimation accuracy. Principle of recursive least square method is explained in [17]. For a given system $y=\boldsymbol{\varphi} \cdot \boldsymbol{\theta}$, where $y$ denote the output vector and $\boldsymbol{\varphi}$ and $\boldsymbol{\theta}$ denote the data matrix and the parameter vector, separately. The implementation process is as follows:

$$
\begin{aligned}
& U_{o}=U_{o c}+I R_{o}+U_{p}, \\
& \dot{U}_{o}=\dot{U}_{o c}+\dot{I} R_{o}+\dot{U}_{p} \text {, } \\
& \dot{U}_{p}=\frac{I}{C_{p}}+\frac{-U_{p}}{R_{p} C_{p}}, \\
& U_{o, k}-U_{o, k-1}=\left(I_{k}-I_{k-1}\right) \cdot R_{o}+T \\
& \cdot\left(\frac{I_{k}}{C_{p}}-\frac{U_{o, k}-U_{o c, k}-I_{k} R_{o}}{R_{p} C_{p}}\right) \text {, } \\
& \left(1+\frac{T}{R_{p} C_{p}}\right) U_{o, k}=U_{o, k-1}+\left(R_{o}+\frac{T}{C_{p}}+\frac{T \cdot R_{o}}{R_{p} C_{p}}\right) \\
& \cdot I_{k}-I_{k-1} \cdot R_{o}+\frac{V_{o c}}{R_{p} C_{p}} T \text {, } \\
& U_{o, k}=\frac{R_{p} C_{p}}{R_{p} C_{p}+T} U_{o, k-1} \\
& +\frac{R_{o} R_{p} C_{p}+T\left(R_{o}+R_{p}\right)}{R_{p} C_{p}+T} I_{k} \\
& -\frac{R_{o} R_{p} C_{p}}{R_{p} C_{p}+T} I_{k-1} \\
& +\frac{U_{o c, k}}{R_{p} C_{p}+T} T \text {, } \\
& U_{o, k}=\theta_{1} \cdot U_{o, k-1}+\theta_{2} \cdot I_{k}+\theta_{3} \cdot I_{k-1} \\
& +\theta_{4} \text {, }
\end{aligned}
$$

$$
\begin{aligned}
\boldsymbol{\varphi}_{k} & =\left[\begin{array}{llll}
U_{o, k-1} & I_{k} & I_{k-1} & 1
\end{array}\right], \\
\boldsymbol{\theta}_{k} & =\left[\begin{array}{llll}
\theta_{1} & \theta_{2} & \theta_{3} & \theta_{4}
\end{array}\right]^{T} .
\end{aligned}
$$

Formula (13) describes the fundamental circuit relationship of first-order RC battery model. In formulas (14)(16), $T$ is sampling interval, as $T$ is very small, so $U_{o c, k} \approx$ $U_{o c, k-1}$, and resistance and capacity can be deduced by (18). Besides, traditional piecewise linear function relationship between SOC and OCV cannot be identified online over the whole range of SOC. Moreover, as open circuit voltage keeps strictly monotone increasing relationship with SOC, thus we refer to the new SOC-OCV functional model expressed in formula (19) which is proposed in [18]. Besides, this function model also applies to $\mathrm{Li}(\mathrm{NiCoMn}) \mathrm{O}_{2}$ batteries; therefore, it is reasonable to adopt this model for online identification of open circuit curve. Take the SOC definition into formula (20) and assume $s(0)=0$. Supposing that resistance and capacitance have been obtained through RLS identification and (20) may take formula (19) then coefficient of OCV function may be obtained through recursive least square method. Formula (21) also clarifies the fundamental circuit relationship of first-order RC battery model and formula (22) is the discretization of formula (21). The detailed solution procedure is as formula (22) to formula (25). Consider

$$
\begin{aligned}
& U_{o c}=a+b \cdot(-\ln s)^{2.1}+c \cdot s+d \cdot e^{s} \quad(s=\mathrm{SOC}), \\
& s(t)=s(0)+\frac{\int i d t}{Q}=s(0)+\frac{q(t)}{Q}, \\
& U_{o}-I R_{o}=U_{1}=U_{o c}+U_{p}, \\
& \dot{U}_{p}=-\frac{U_{p}}{R_{p} C_{p}}+\frac{I}{C_{p}}, \\
& \dot{U}_{1}=\dot{U}_{p}+\dot{U}_{o c}=\dot{U}_{o c}-\frac{U_{1}-U_{o c}}{R_{p} C_{p}}+\frac{I}{C_{p}}, \\
& \frac{U_{1, k}-U_{1, k-1}}{T}=\frac{U_{o c, k}-U_{o c, k-1}}{T}+\frac{I_{k}}{C_{p}}-\frac{U_{1, k}-U_{o c, k}}{R_{p} C_{p}},
\end{aligned}
$$




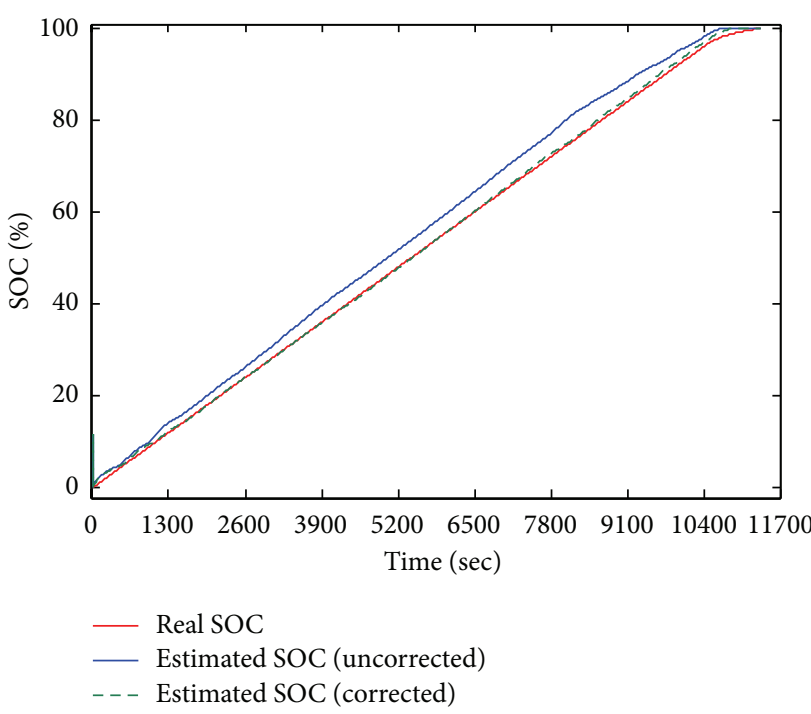

(a)

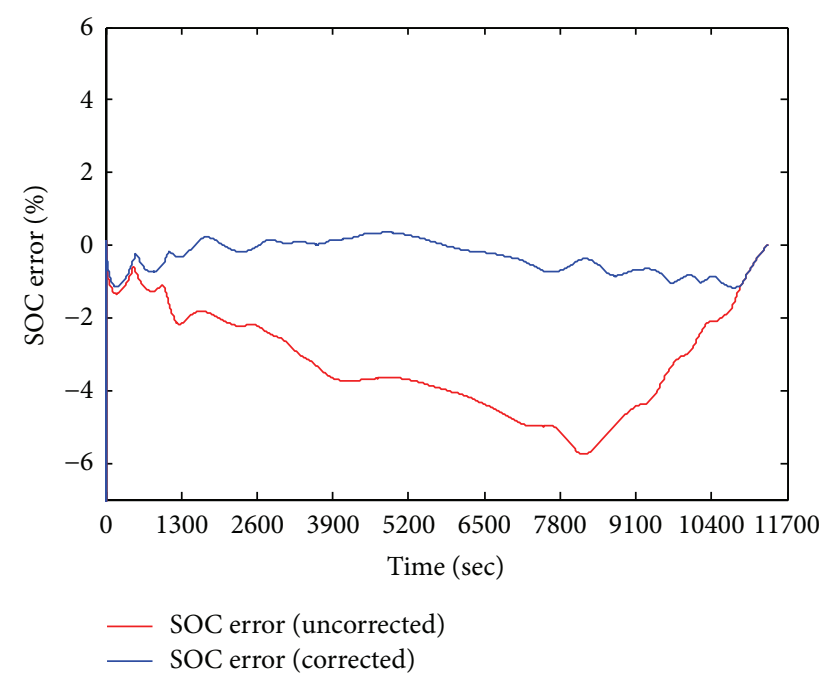

(b)

FIGURE 5: SOC estimation results before and after correction of the internal resistance.

$$
\begin{aligned}
U_{1, k}- & U_{1, k-1}+\frac{T \cdot U_{1, k}}{R_{p} C_{p}} \\
= & U_{o c, k}+\frac{T \cdot U_{o c, k}}{R_{p} C_{p}}-U_{o c, k-1}+T \cdot \frac{I_{k}}{C_{p}}, \\
U_{1, k} & \\
= & \frac{R_{p} C_{p}}{R_{p} C_{p}+T} \cdot U_{1, k-1}+\frac{T \cdot R_{p}}{R_{p} C_{p}+T} \cdot I_{k}+b \\
& \cdot\left[\left(-\ln \frac{q_{k}}{Q}\right)^{2.1}-\left(-\ln \frac{q_{k-1}}{Q}\right)^{2.1}\right]+\frac{c}{Q} \\
& \cdot\left(q_{k}-q_{k-1}\right)+d \cdot\left(e^{q_{k} / Q}-e^{q_{k-1} / Q}\right) \\
& +\frac{T \cdot a}{R_{p} C_{p}+T}, \\
U_{1, k} & \theta_{1} \cdot U_{1, k-1}+\theta_{2} \cdot I_{k}+\theta_{3} \\
& \cdot\left[\left(-\ln \frac{q_{k}}{Q}\right)^{2.1}-\left(-\ln \frac{q_{k-1}}{Q}\right)^{2.1}\right]+\theta_{4} \\
& {\left[q_{k}-q_{k-1}\right)+\theta_{5} \cdot\left(e^{q_{k} / Q}-e^{q_{k-1} / Q}\right)+\theta_{6} . }
\end{aligned}
$$

Considering the real situation that the battery gets old but model parameters are not updated timely, simulation results before and after the parameter correction will be presented in following figures to validate the theoretical results. Comparison of two estimation results which only update the OCV-SOC curve but do not change the internal resistance of aged battery under CCCV charging mode at $25^{\circ} \mathrm{C}$ is shown in Figure 5. It can be observed that SOC error with uncorrected, namely, not updated, internal resistance is larger and the maximum error reaches about 6\%. However, SOC estimation error is greatly reduced and the maximum error is within $1.5 \%$ after parameter correction. On the contrary, Figure 6 presents comparison results for the same battery in which only OCV-SOC curves are amended. Maximum error reduces from $3.5 \%$ to $1 \%$ and the average error gets smaller. Comparing Figures 5 and 6, the resistance error has greater impact on SOC estimation precision in this case due to extent of change on battery resistance and OCV caused by aging is different. Simulation validation results at different temperatures can get similar conclusions and all results will not be enumerated here. All these verify the conclusion that accuracy of the model parameters can improve the precision of the SOC estimation.

\section{Validation and Discussions}

4.1. Hardware-in-Loop Experimental Platform. The real-time current/voltage acquisition experimental platform is illustrated in Figure 7. Arbin BTS2000 is the charger to charge/ discharge the battery and the battery is laid in the thermostat to maintain constant temperature. BMS includes current/voltage acquisition board and CAN-TCP conversion card. The computer is a monitor to control the experiments, obtaining voltage/current data from sampling board, and carry out the simulation under MATLAB/Simulink. Sampling frequency of the voltage acquisition board is less than $30 \mathrm{~ms}$, voltage sampling error is less than $10 \mathrm{mV}$, and current sampling precision is less than $1 \%$. The interface between acquisition board and external communication is CAN buses and CAN-TCP conversion card is added to receive the CAN bus data frame. The S-function module is utilized to call the CAN-TCP conversion card driver program and return current/voltage data to Simulink space. Besides, real-time sync module is used for synchronizing the simulation clock with real-time clock. All modules in platform work together to 


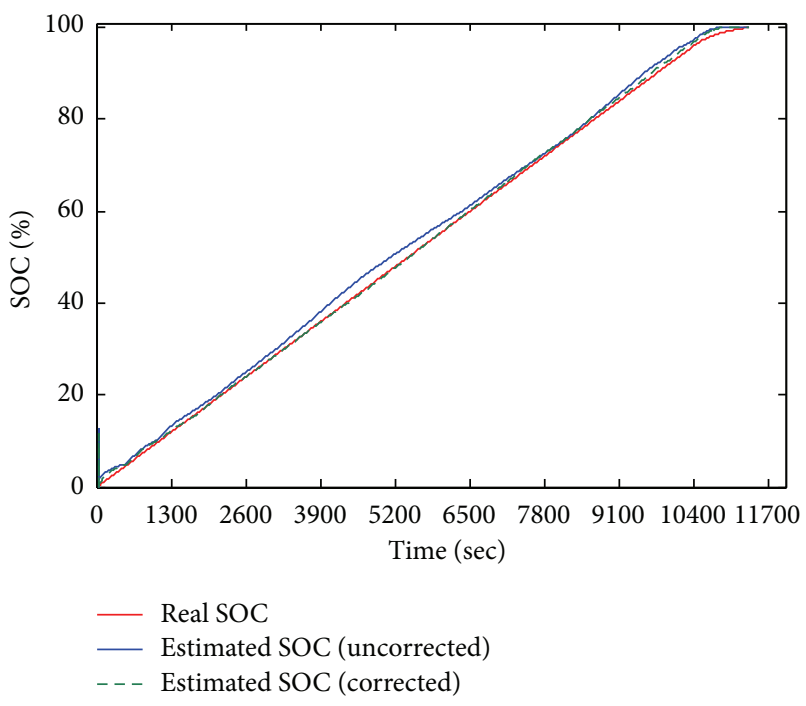

(a)

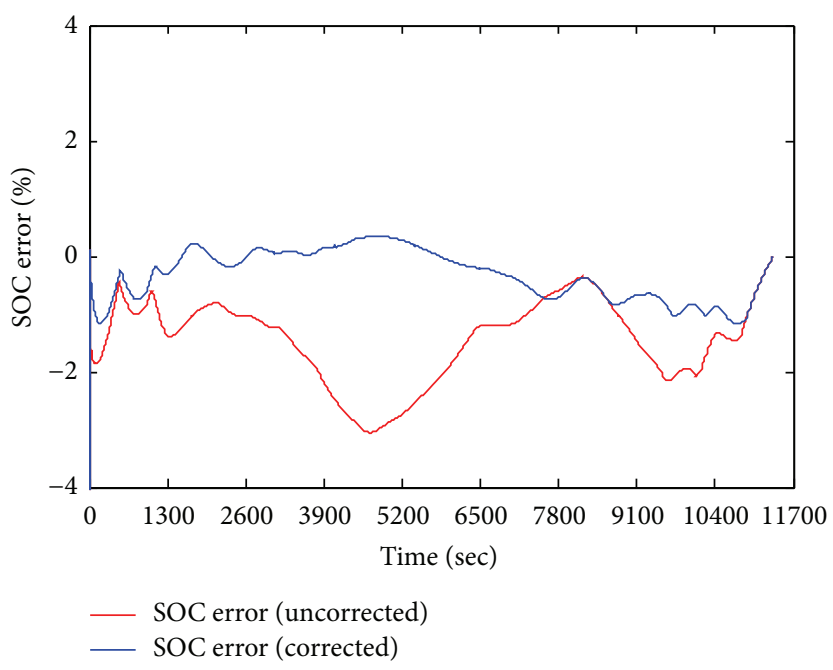

(b)

FIGURE 6: SOC estimation results before and after the correction of the OCV-SOC curve.

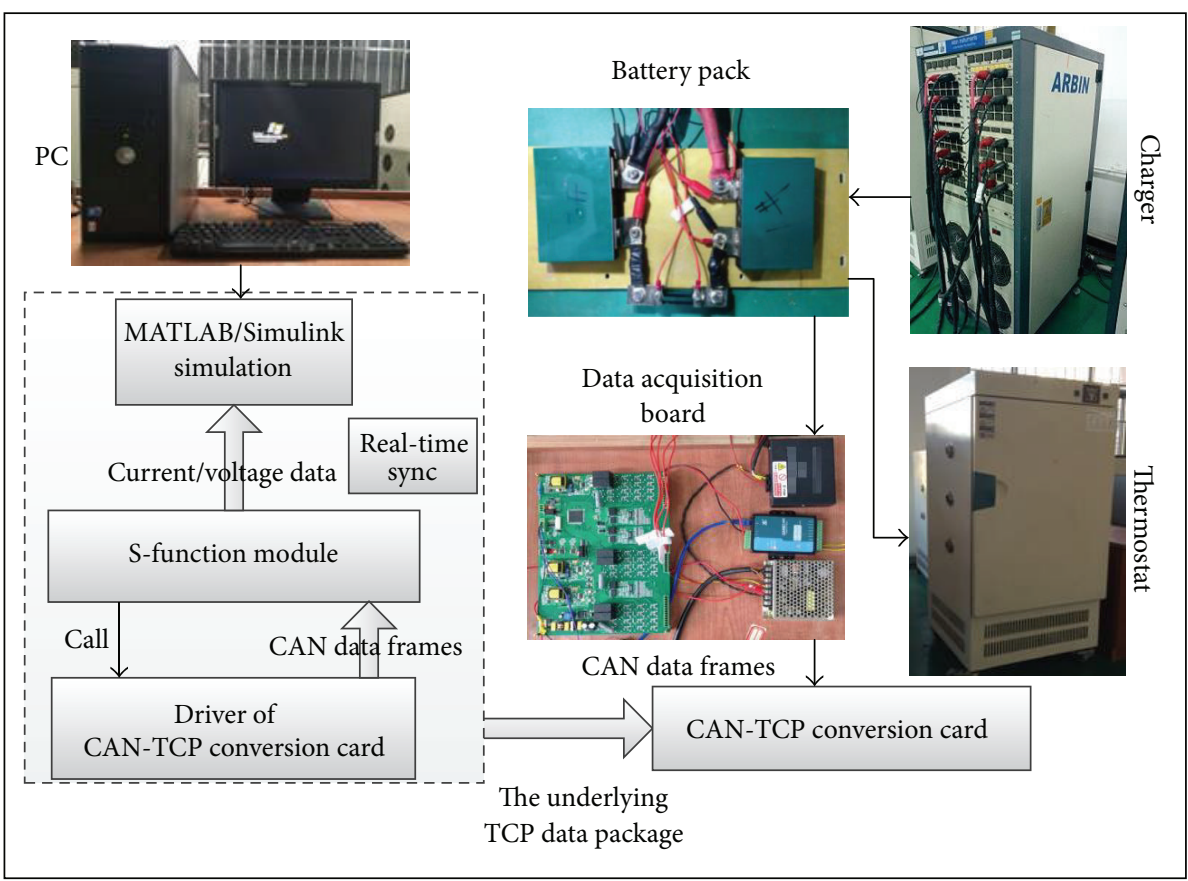

Figure 7: Experimental platform.

ensure that the current and voltage data flows into the simulation space and online real-time simulation is achieved eventually.

4.2. Experimental Results and Discussions. We take two series-connected $\mathrm{Li}(\mathrm{NiCoMn}) \mathrm{O}_{2}$ batteries with the capacity of $28 \mathrm{Ah}$ as test objects. Considering that online identification results of battery model parameters may lead to relatively larger model error when at lower SOC, which leads to SOC estimation error based on PI observer method increases at low SOC, therefore, PI observer and ampere hour integration method are combined to obtain SOC for a battery pack. Besides, this combination also reduces computation cost to some extent. The transforming SOC value from PI observer to ampere hour integration method can be decided by the practical experience. For battery model, resistance and capacitor parameters can be obtained under a certain SOC. We can get some regular patterns that these battery parameters have fluctuation change under low SOC, and there would be some SOC value that can be defined as transforming SOC value. During discharging of the battery, when estimated SOC value based on PI observer is above $40 \%$, we take the observer 


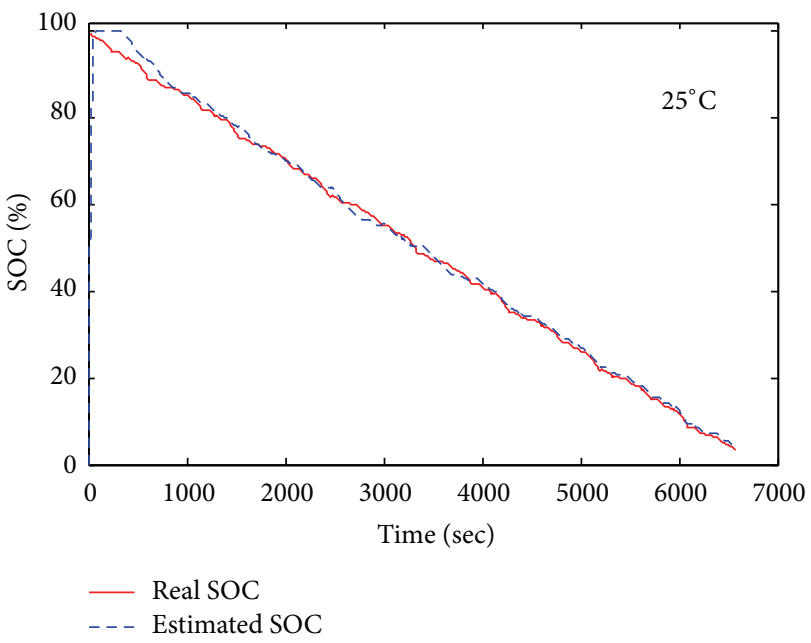

(a)

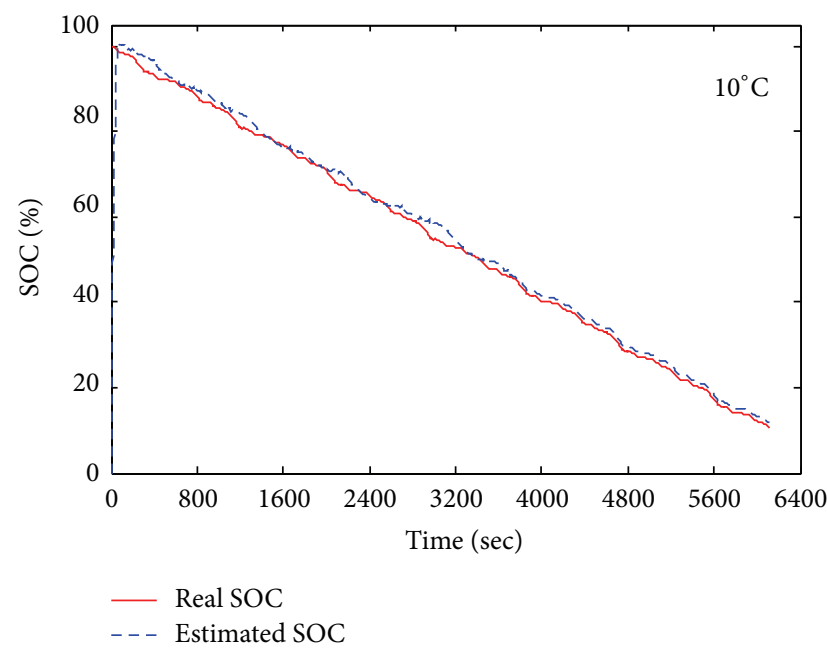

(c)

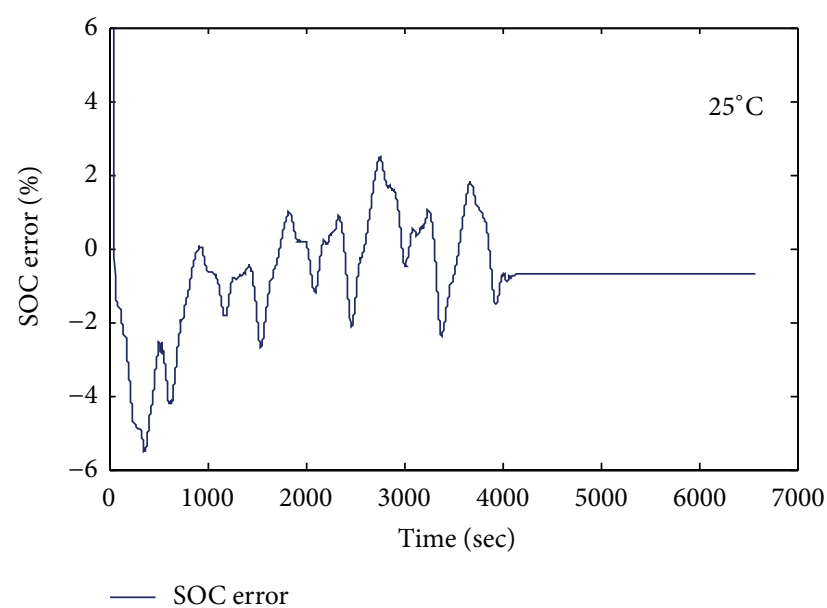

(b)

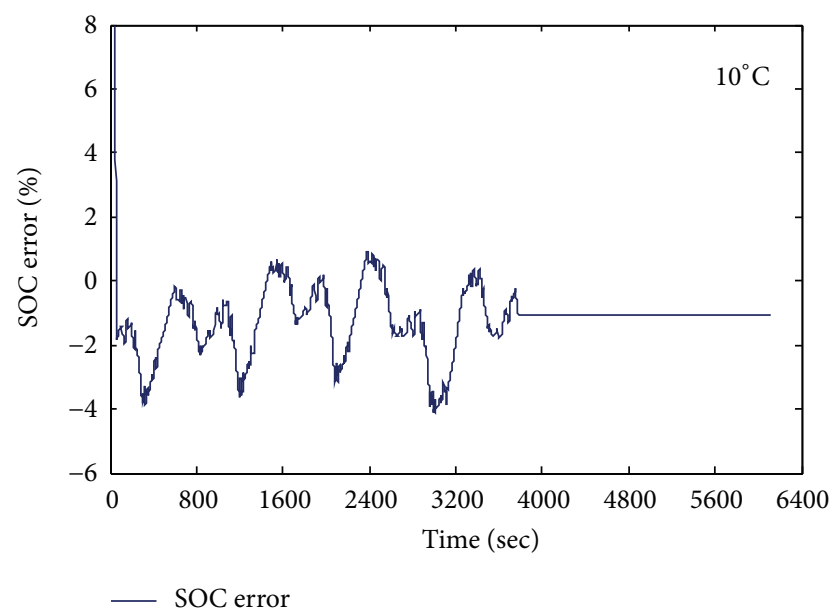

(d)

FIGURE 8: Real-time SOC estimation results under BJDST condition at different temperature.

estimation value as SOC output; however, as it is below $40 \%$, ampere hour integration value with the initial value of $40 \%$ will be regarded as the SOC output. Figures 8 and 9 are SOC estimation results with this method under different temperature, dynamic working conditions, and initial SOC value, respectively.

Because the low temperature will have a greater impact on characteristics of batteries, we choose low temperature $10^{\circ} \mathrm{C}$ and room temperature $25^{\circ} \mathrm{C}$ to do comparison and verification experiments. Figure 8 shows SOC online estimation results under BJDST current conditions at $25^{\circ} \mathrm{C}$ and $10^{\circ} \mathrm{C}$, respectively. Figures 8 (b) and 8 (d) are corresponding error curves. As shown in Figure 8, maximum estimation error is within $5 \%$ and average error is $2 \%$. Besides, SOC error is relatively higher at $10^{\circ} \mathrm{C}$ because of low temperature influence. On the whole, this combination method notably increases estimation precision during the whole SOC interval. SOC calculation results with $10 \%$ and $30 \%$ divergences of SOC initial value between two cells are described in Figures 9(a) and 9(c) separately. Figures 9(b) and 9(d) are also corresponding error curves. It is noted that this combination method proposed in the paper is also applicable to the case with inconsistencies between the cells in a battery pack. Additionally, the precision is within $3 \%$; thus this method is reliable.

\section{Conclusions}

Recognizing that SOC estimation is affected by battery model parameters, temperature, operation conditions, and cell inconsistency, the estimation error sensitivity for model parameters is firstly qualified and analyzed, concluding that the SOC estimation error resulting from the internal resistance inaccuracy will be enlarged as charge/discharge current increase; the effect of polarization capacity on SOC estimation can be neglected since it is involved with transient error, and the SOC-OCV mapping is the most crucial function in SOC estimation. The SOC estimation method for a lithium-ion battery pack taking error sensitivity analysis and cell inconsistency into consideration is developed, and the recursive parameters update method is also presented. Using 


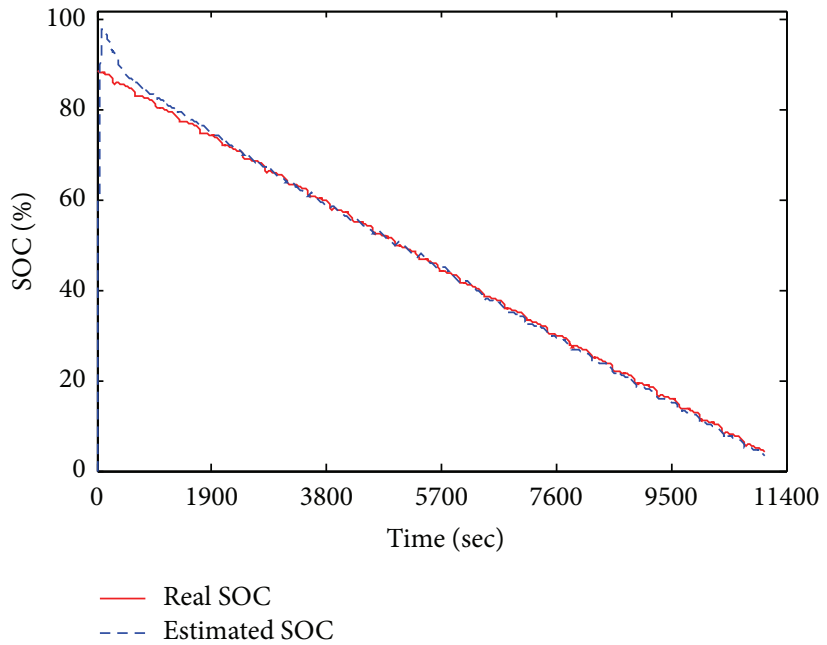

(a)

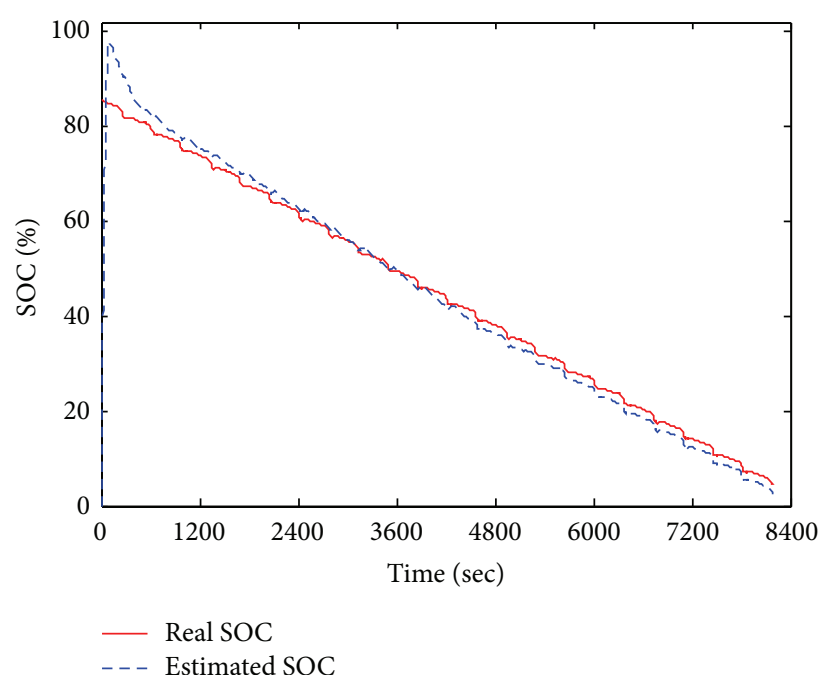

(c)

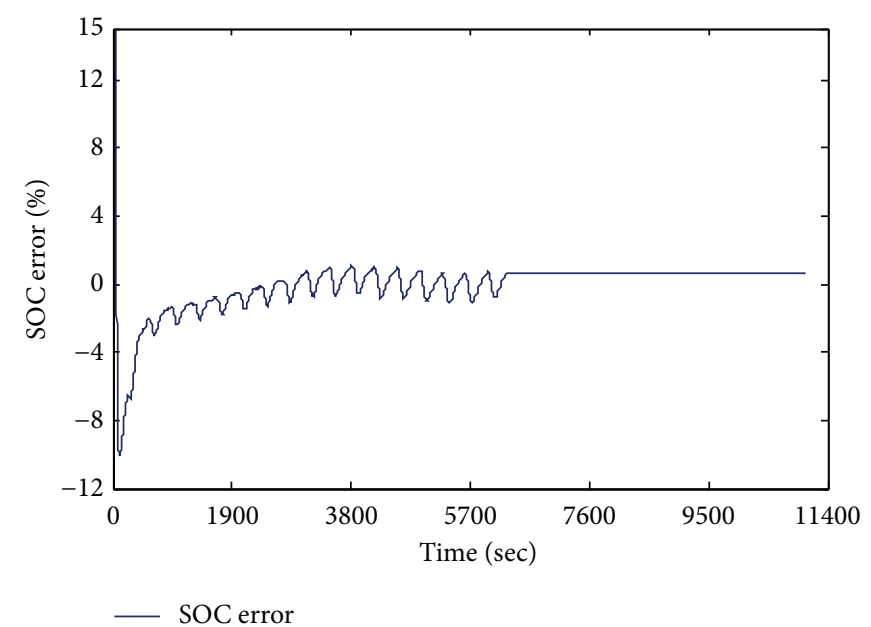

(b)

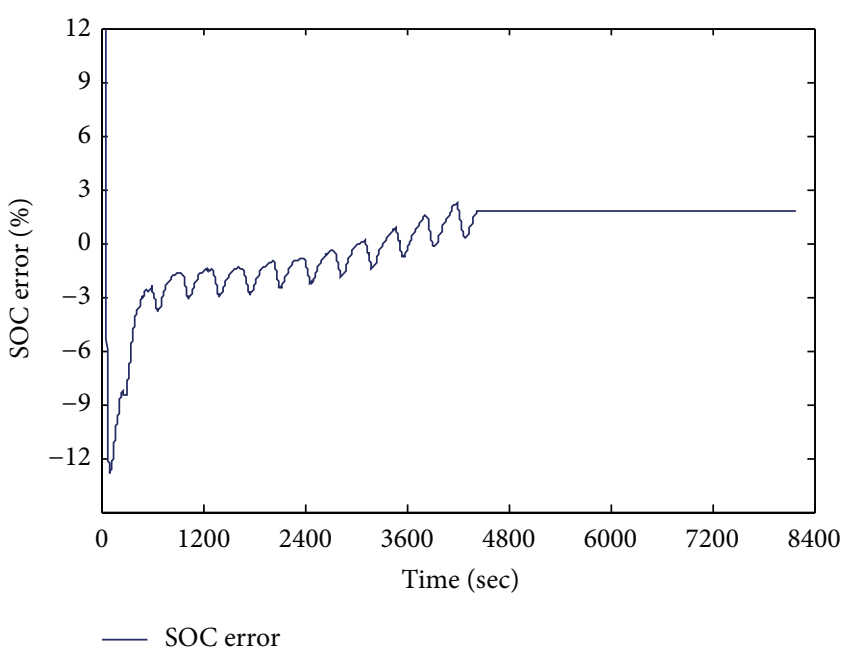

(d)

FIGURE 9: Real-time SOC estimation results under DST condition at $25^{\circ} \mathrm{C}$ with different SOC initial value.

the hardware-in-loop simulation platform, the experimental results at various dynamic test conditions, temperatures, and initial SOC difference between two cells demonstrate that the estimation error can be controlled within $4 \%$, verifying the efficacy of the proposed method.

\section{Conflict of Interests}

The authors declare no conflict of interests.

\section{Authors' Contribution}

The theoretical analysis, experiments, and simulation verification were done by Ting Zhao. Besides, this work was also performed under the advisement from Jiuchun Jiang and Caiping Zhang, who also revised the paper critically. Kai Bai and $\mathrm{Na} \mathrm{Li}$ gave some useful information and suggestions for this paper.

\section{Acknowledgment}

This work was supported by the National Natural Science Foundation of China Project (51477009).

\section{References}

[1] W. Zhenpo and S. Fengchun, Electric Vehicle Power Battery System and Its Applications, Machinery Industry Press, Beijing, China, 2012.

[2] J. Li, J. Klee Barillas, C. Guenther, and M. A. Danzer, "A comparative study of state of charge estimation algorithms for $\mathrm{LiFePO}_{4}$ batteries used in electric vehicles," Journal of Power Sources, vol. 230, pp. 244-250, 2013.

[3] G. L. Plett, "Extended Kalman filtering for battery management systems of LiPB-based HEV battery packs-part 1. Background," Journal of Power Sources, vol. 134, no. 2, pp. 252-261, 2004.

[4] X. Hu, F. Sun, and Y. Zou, "Comparison between two modelbased algorithms for Li-ion battery SOC estimation in electric 
vehicles," Simulation Modelling Practice and Theory, vol. 34, pp. 1-11, 2013.

[5] W.-Y. Chang, "Estimation of the state of charge for a LFP battery using a hybrid method that combines a RBF neural network, an OLS algorithm and AGA," International Journal of Electrical Power \& Energy Systems, vol. 53, no. 1, pp. 603-611, 2013.

[6] J. Gao and H. He, "Comparison of nonlinear filtering methods for estimating the state of charge of $\mathrm{Li}_{4} \mathrm{Ti}_{5} \mathrm{O}_{12}$ lithium-ion battery," Mathematical Problems in Engineering, In press.

[7] H. Dai, X. Wei, Z. Sun, J. Wang, and W. Gu, "Online cell SOC estimation of Li-ion battery packs using a dual time-scale Kalman filtering for EV applications," Applied Energy, vol. 95, pp. 227-237, 2012.

[8] X. Liu, Y. He, and Z. Chen, "State-of-charge estimation for power Li-ion battery pack using Vmin-EKF," in Proceedings of the 2nd International Conference on Software Engineering and Data Mining (SEDM '10), pp. 27-31, Chengdu, China, June 2010.

[9] G. L. Plett, "Extended Kalman filtering for battery management systems of LiPB-based HEV battery packs-part 2. Modeling and identification," Journal of Power Sources, vol. 134, no. 2, pp. 262-276, 2004.

[10] Z. Chen, Y. Fu, and C. C. Mi, "State of charge estimation of lithium-ion batteries in electric drive vehicles using extended Kalman filtering," IEEE Transactions on Vehicular Technology, vol. 62, no. 3, pp. 1020-1030, 2013.

[11] Y. Zheng, M. Ouyang, L. Lu et al., "Cell state-of-charge inconsistency estimation for $\mathrm{LiFePO}_{4}$ battery pack in hybrid electric vehicles using mean-difference model," Applied Energy, vol. 111, pp. 571-580, 2013.

[12] Z. Cheng, J. Lv, Y. Liu, and Z. Yan, "Estimation of state of charge for lithium-ion battery based on finite difference extended Kalman filter," Journal of Applied Mathematics, vol. 2014, Article ID 348537, 10 pages, 2014.

[13] C. Truchot, M. Dubarry, and B. Y. Liaw, "State-of-charge estimation and uncertainty for lithium-ion battery strings," Applied Energy, vol. 119, pp. 218-227, 2014.

[14] M. A. Roscher, O. S. Bohlen, and D. U. Sauer, "Reliable state estimation of multicell lithium-ion battery systems," IEEE Transactions on Energy Conversion, vol. 26, no. 3, pp. 737-743, 2011.

[15] T. Zhao, J. Jiang, C. Zhang, L. Zheng, and F. Wen, "Error analysis of SOC estimation based on PI observer," in Proceedings of the IEEE Transportation Electrification Conference and Expo (ITEC '14), pp. 1-5, Beijing, China, September 2014.

[16] O. Katsuhiko, Modern Control Engineering, Publishing House of Electronics Industry, Beijing, China, 2011.

[17] T. Xia, G. Xiong, and F. Li, System Identification: Least Square Method, Publishing House of Tsinghua University, Beijing, China, 1983.

[18] C. Zhang, L. Y. Wang, X. Li, W. Chen, G. G. Yin, and J. Jiang, "Robust and adaptive estimation of state of charge for lithiumion batteries," IEEE Transactions on Industrial Electronics, vol. 62, no. 8, pp. 4948-4957, 2015. 


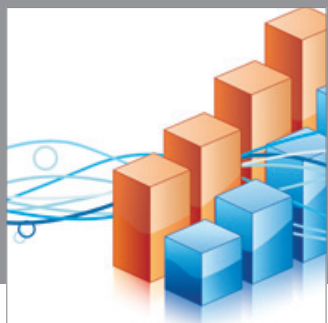

Advances in

Operations Research

mansans

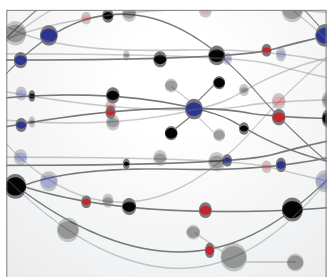

The Scientific World Journal
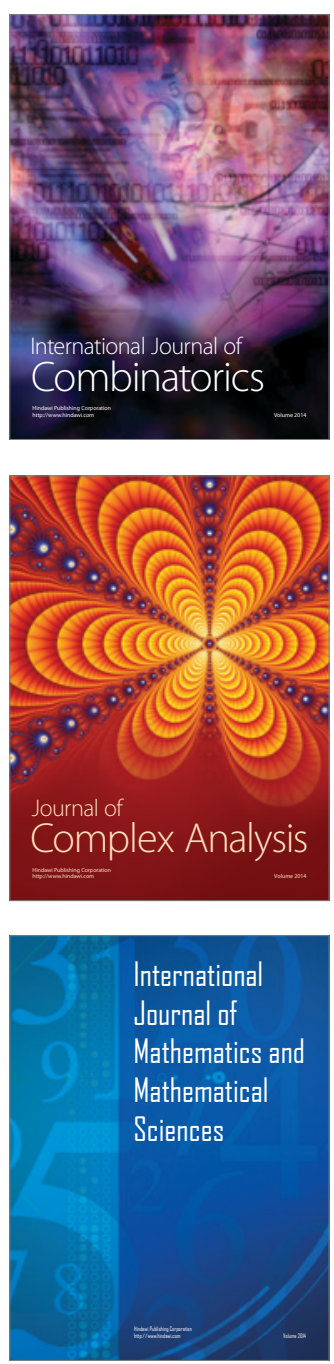
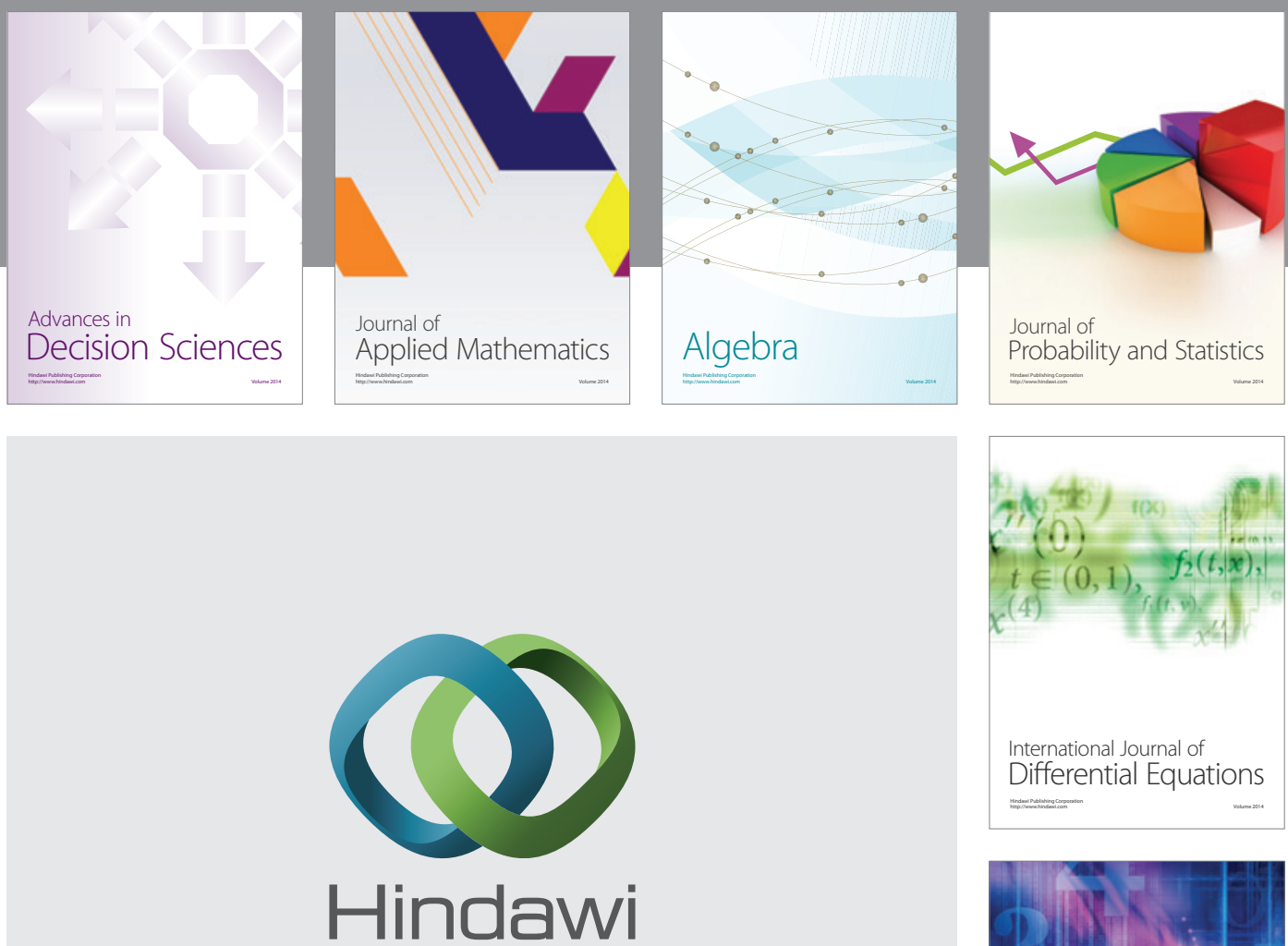

Submit your manuscripts at http://www.hindawi.com
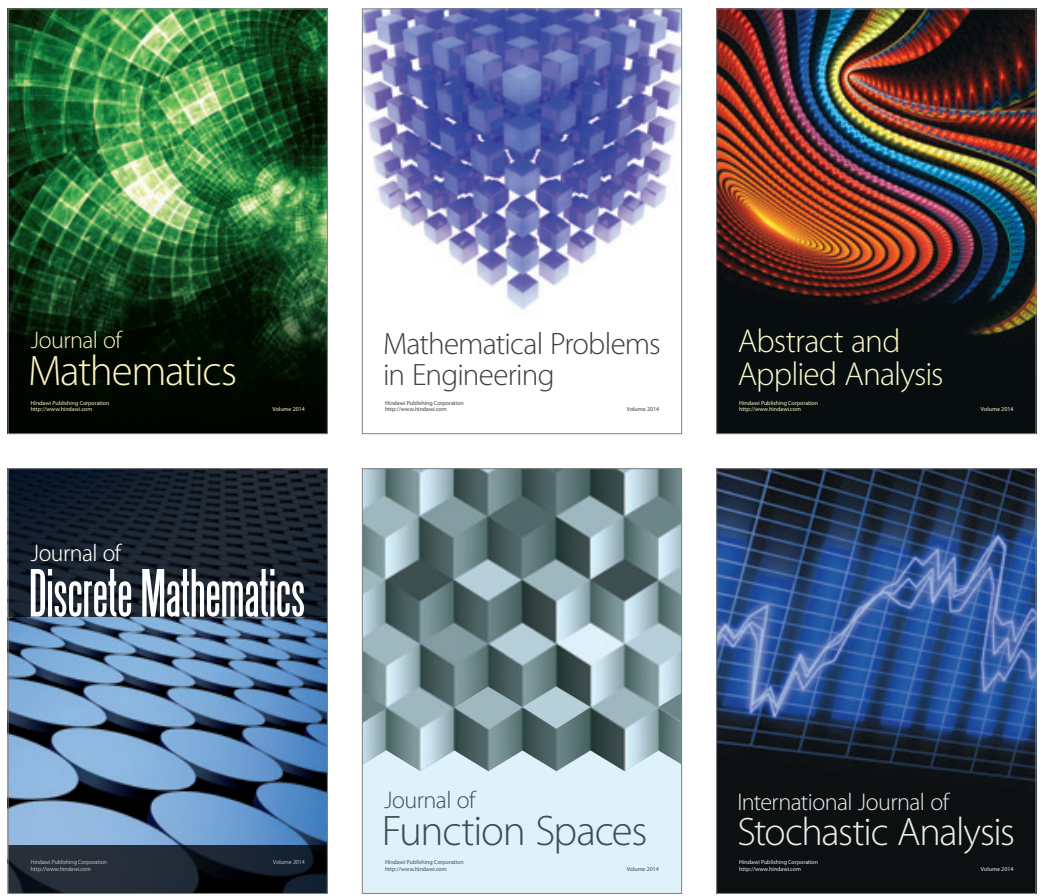

Journal of

Function Spaces

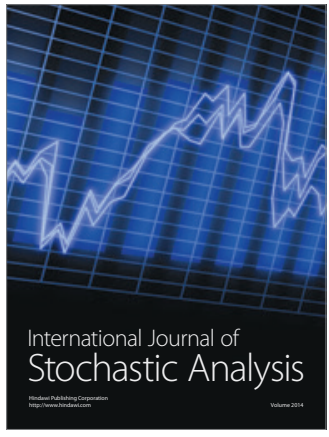

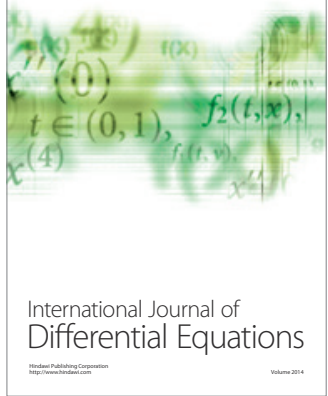
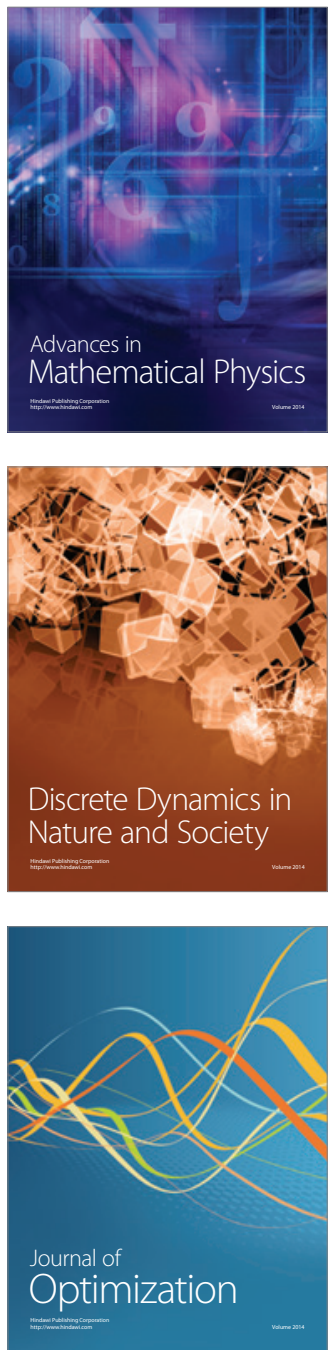